\title{
A Rare Case Report of Intravenous Leiomyomatosis with Intracardiac Extension: Role of Transesophageal Echocardiography
}

\author{
${ }^{1}$ Srinath Damodaran, ${ }^{2}$ Krishna Prasad Gourav, ${ }^{3}$ Rashmi Bagga, ${ }^{4}$ Pankaj Aggarwal, ${ }^{5}$ Aruna Singh, ${ }^{6}$ Gurpinder Singh \\ Ghotra, ${ }^{7}$ Sunder Lal Negi, ${ }^{8}$ Parveen Negi
}

\begin{abstract}
Uterine leiomyoma is a common benign tumor that arises from the overgrowth of smooth muscle and connective tissue in the uterus. Transesophageal echocardiography is a very useful diagnostic modality in the intraoperative period as it provides us real time imaging. We report a case of uterine leiomyoma with intracaval and right atrial extension that TEE was helpful in the intraoperative management.
\end{abstract}

Keywords: Cardiac extension, Transesophageal echocardiography, Uterine leiomyoma.

How to cite this article: Damodaran S, Gourav KP, Bagga R, Aggarwal P, Singh A, Ghotra GS, Negi SL, Negi P. A Rare Case Report of Intravenous Leiomyomatosis with Intracardiac Extension: Role of Transesophageal Echocardiography. J Perioper Echocardiogr 2018;6(2):60-63.

Source of support: Nil

Conflict of interest: None

\section{INTRODUCTION}

Leiomyoma is a benign smooth muscle cell tumor of uterine origin that may extend into the pelvic veins and the inferior vena cava. ${ }^{1-4}$ The tumor is mainly confined to pelvic veins but it can spread through the inferior vena

1,2,5Senior Resident, ${ }^{3}$ Professor, ${ }^{4,7}$ Assistant Professor, ${ }^{6}$ Fellow, ${ }^{8}$ Junior Resident

${ }^{1}$ Department of Cardiac Anesthesiology, Postgraduate Institute of Medical Education and Research, Chandigarh, India

${ }^{2}$ Department of Anesthesia, Postgraduate Institute of Medical Education and Research, Chandigarh, India

${ }^{3,5}$ Department of Obstetrics and Gynecology, Postgraduate Institute of Medical Education and Research, Chandigarh, India

${ }^{4}$ Department of Cardiothoracic and Vascular Surgery, Postgraduate Institute of Medical Education and Research, Chandigarh, India

6-8 Department of Anesthesia and Intensive Care, Postgraduate Institute of Medical Education and Research, Chandigarh, India

Corresponding Author: Sunder Lal Negi, Assistant Professor, Department of Anesthesia and Intensive Care, Postgraduate Institute of Medical Education and Research, Chandigarh, India, e-mail: dr.sundernegi@gmail.com cava (IVC) to the right atrium (RA), when it is referred to as intravenous leiomyomatosis. The extension of the tumor into the right-sided cardiac chambers is rarely seen. In most patients intravenous leiomyomatosis presents in the 4th and the 5 th decade of life. We report a case of uterine leiomyoma with intracaval and right atrial extension, in which transesophageal echocardiography was used as a monitoring device for perioperative management.

\section{CASE DESCRIPTION}

A 35-year-old female presented with easy fatigability and lower abdominal pain. On evaluation, she was found to have a lower abdomino pelvic mass. Biopsy report was suggestive of smooth muscle neoplasm. Contrast enhanced computed tomography (CECT) of the abdomen revealed $11 \times 9 \times 14 \mathrm{~cm}$ enhancing mass arising from the myometrium predominantly on the right side. There was also a central hypodence filling defect seen in the inferior vena cava (IVC) that extended from the right common iliac vein all the way up to the right atrium (RA). The left common iliac vein was normal. Other laboratory parameters were within normal limit. The surgical plan was for a transabdominal hysterectomy and bilateral salpingo-oophorectomy with retrieval of the tumor.

Patient was premedicated with ranitidine $50 \mathrm{mg}$ and alprazolam $0.25 \mathrm{mg}$, the previous night and the morning of surgery. In the operating room (OR), standard monitors were attached to the patient and a $16 \mathrm{G}$ intravenous cannula was secured in the left upper arm. General anesthesia was induced with fentanyl $100 \mu \mathrm{g}$ and propofol $100 \mathrm{mg}$ and intubation was facilitated with vecuronium $6 \mathrm{mg}$. For blood pressure monitoring, an arterial line was secured with a 20G cannula in the left radial artery. A transesophageal echocardiography (TEE) probe was placed in the oesophagus. Additionally, a triple lumen central line was secured in the right internal jugular vein, after confirmation of the guide wire with the TEE to prevent related complications such as arterial cannulation or tumor dislodgement (Fig. 1). 


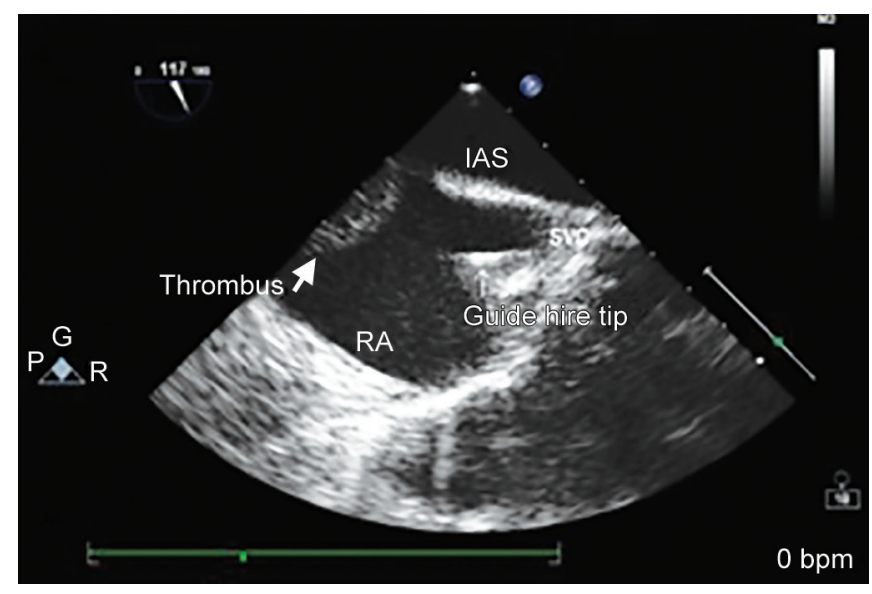

Fig. 1: Mid-esophagealbicaval view showing guidewire tip at upper end of right atrium and thrombus protruding from SVC.

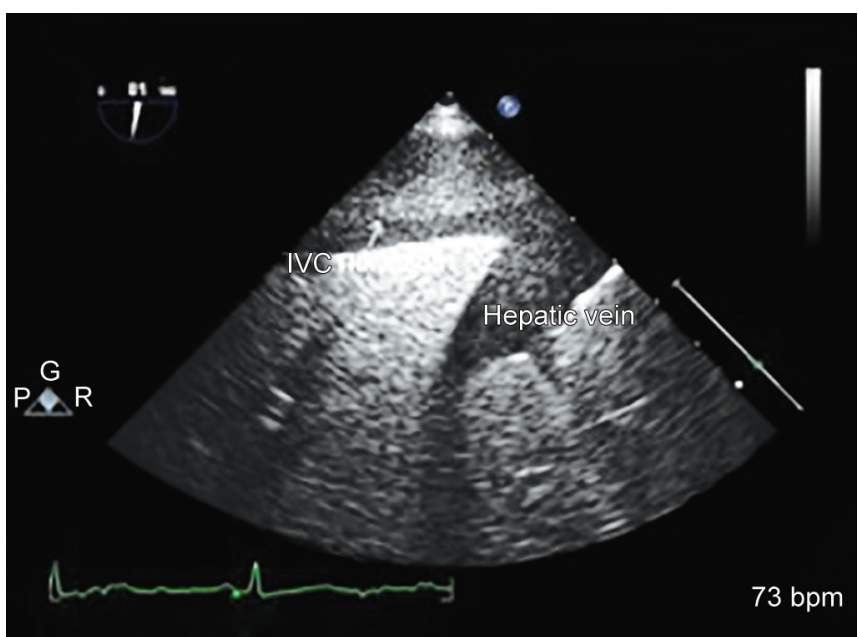

Fig. 3: Transgastric long axis view of IVC with hepatic vein draining into IVC with tumor in IVC.

Anaesthesia was maintained with air, oxygen, isoflurane and fentanyl. TEE revealed a round mass of $3 \times 3 \mathrm{~cm}$ protruding into the right atrium from the IVC (Fig. 2), on tracing down mass was extending from infra hepatic IVC to right atrium. TEE also demonstrated tumor in IVC and was non adherent to vessel wall (Fig. 3) and no tumor in main pulmonary artery, and right pulmonary artery (Fig. 4). Sternotomy was performed and pericardium opened. Transabdominal hysterectomy and bilateral salpingo-oophorectomy was performed through a midline abdominal incision. Suprarenal and infrarenal IVC were dissected, following which the patient was heparinized to achieve activated clotting time of 480 seconds. After aortic and superior vena cava (SVC) cannulation the right atrium was opened and sucker was placed into right atrium to drain blood coming from the IVC and coronary sinus, and partial cardiopulmonary bypass (CPB) was initiated. Upon opening of the right atrium, a round shiny mass protruding from the IVC was visualised (Fig. 5). Suprarenal IVC was looped, venacavotomy was done and mass was retrieved with gentle pull from suprarenal and infrarenal IVC. The

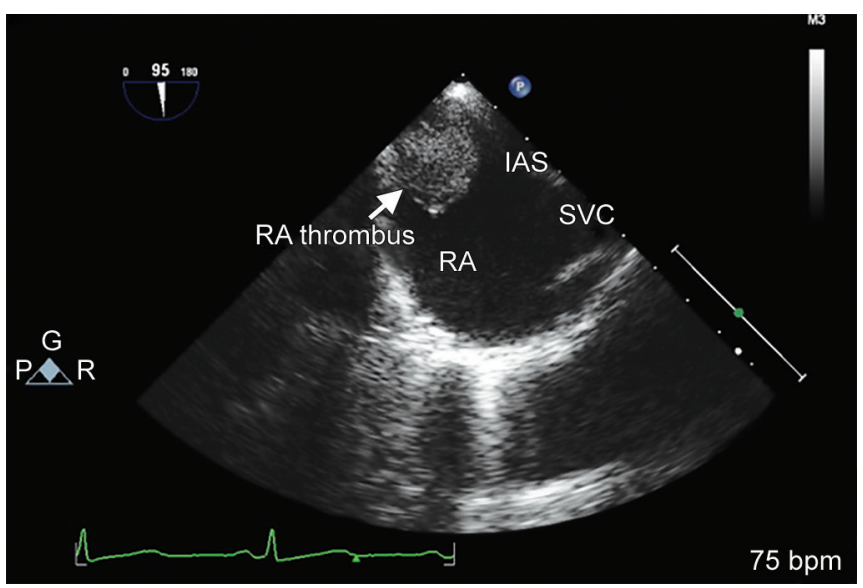

Fig. 2: Mid-esophagealbicaval view showing thrombus protruding from IVC.

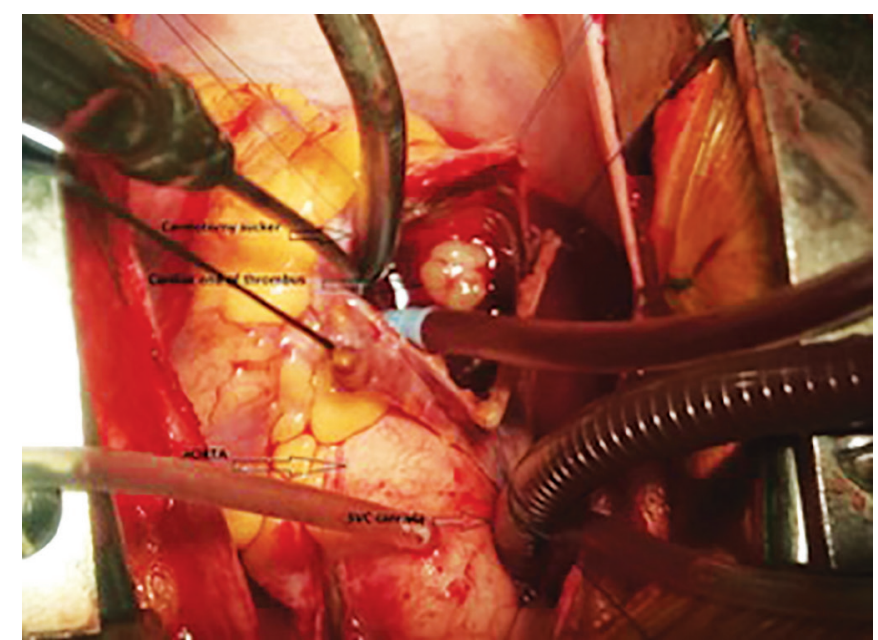

Fig. 4: Opened right atrium showing shiny round thrombus protruding from IVC and cardiotomy sucker in right atrium.

tumor was around $30 \mathrm{~cm}$ long with rounded cardiac end and tapered iliac ends (Fig. 6). A comprehensive TEE exam was performed to rule out potential emboli and residual mass with continuing monitoring throughout the procedure. The IVC was repaired and the right atrium was closed. Patient came off cardiopulmonary bypass without any inotropic support and the total CPB time was 22 minutes. Postprocedure TEE showed no residual mass in the right atrium or the IVC along with normal flow pattern in the pulmonary arteries (Fig. 7). Patient was shifted to the intensive care unit and extubated after 6 hours. After an unremarkable hospital course, the patient was discharged from the hospital on postoperative day six.

\section{DISCUSSION}

Uterine leiomyoma is a common benign tumor of the uterus in women; however, intravenous leiomyomatosis with intracaval and intracardiac extension is a rare phenomenon. ${ }^{1,2}$ Most of the time it is confined to the pelvic veins. Because of its nonspecific clinical 


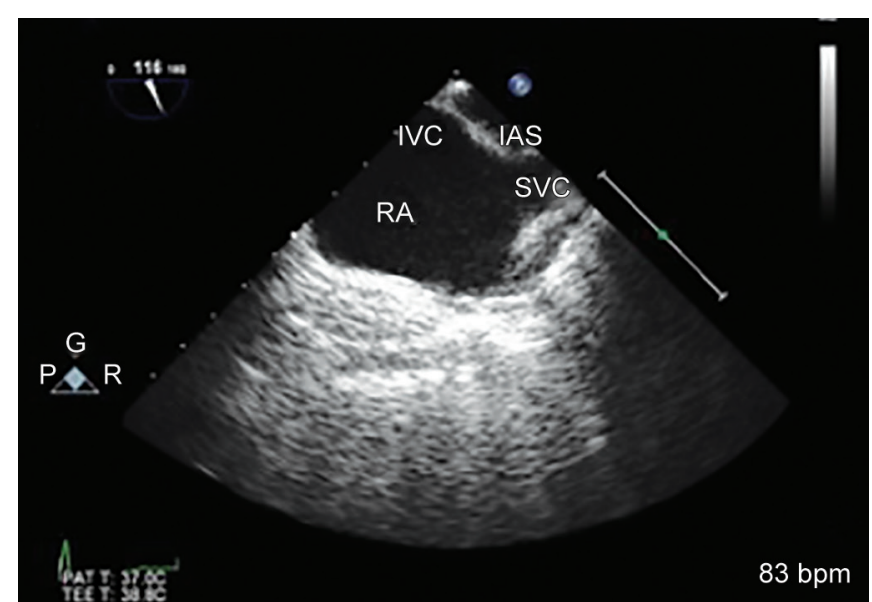

Fig. 5: Mid-esophageal bicaval view showing empty right atrium with SVC and IVC communicating with right atrium.
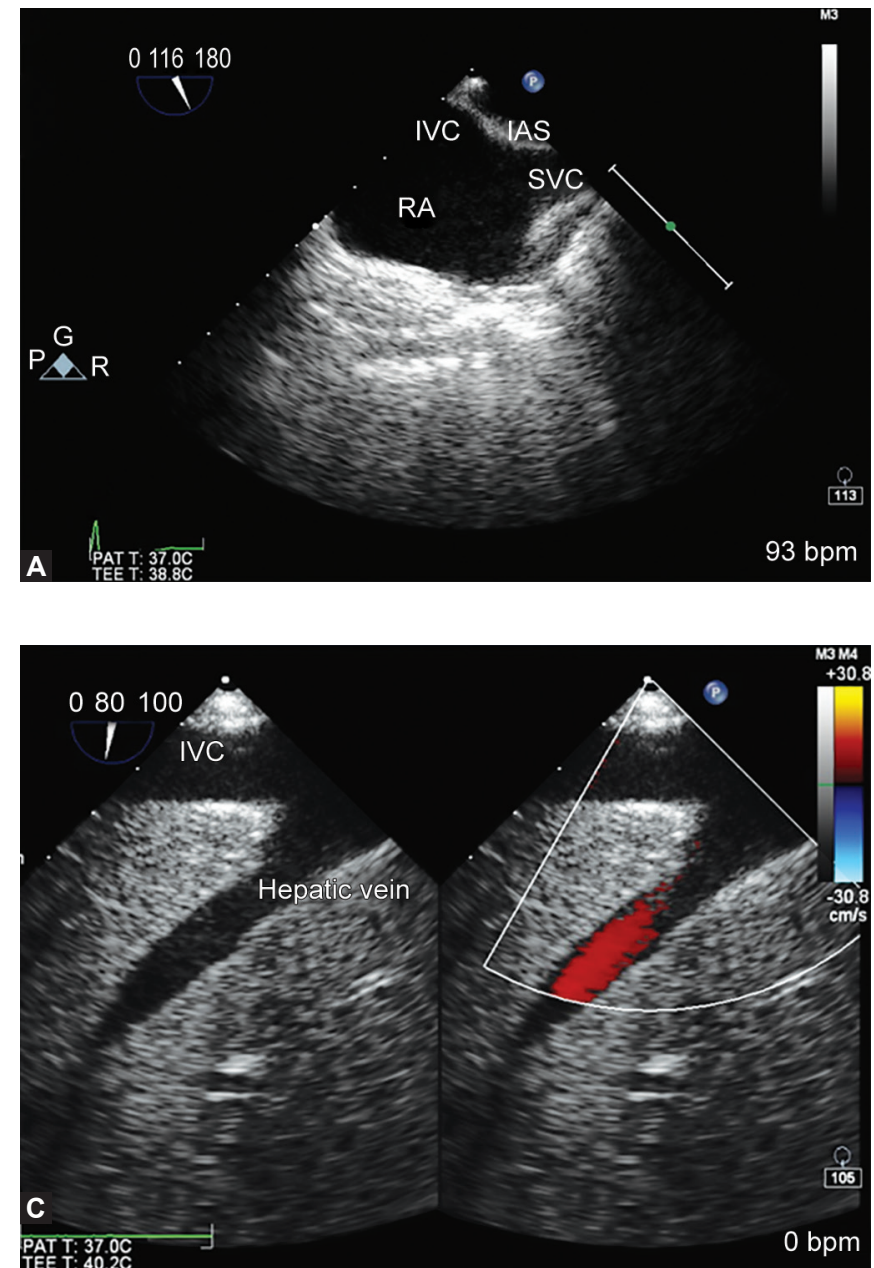

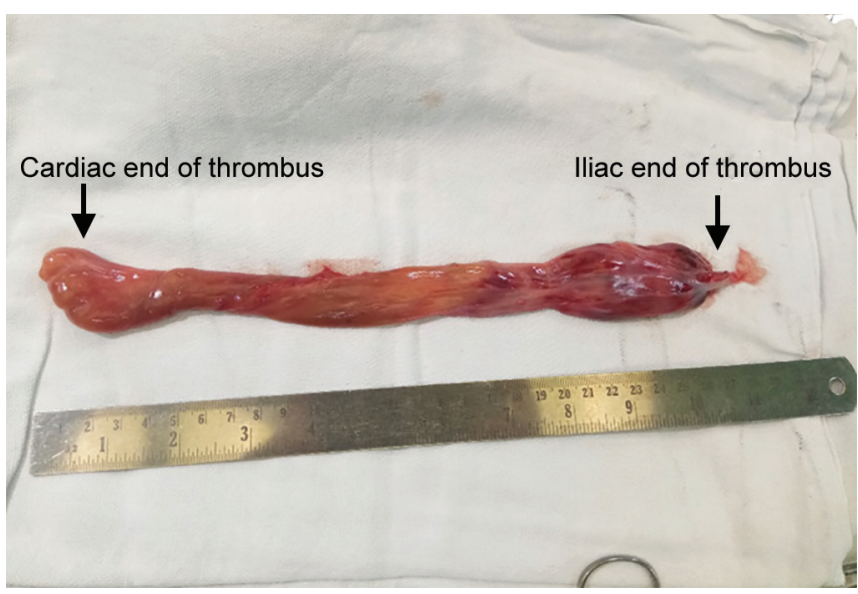

Fig. 6: Distal round cardiac end and proximal tapered iliac end of thrombus.
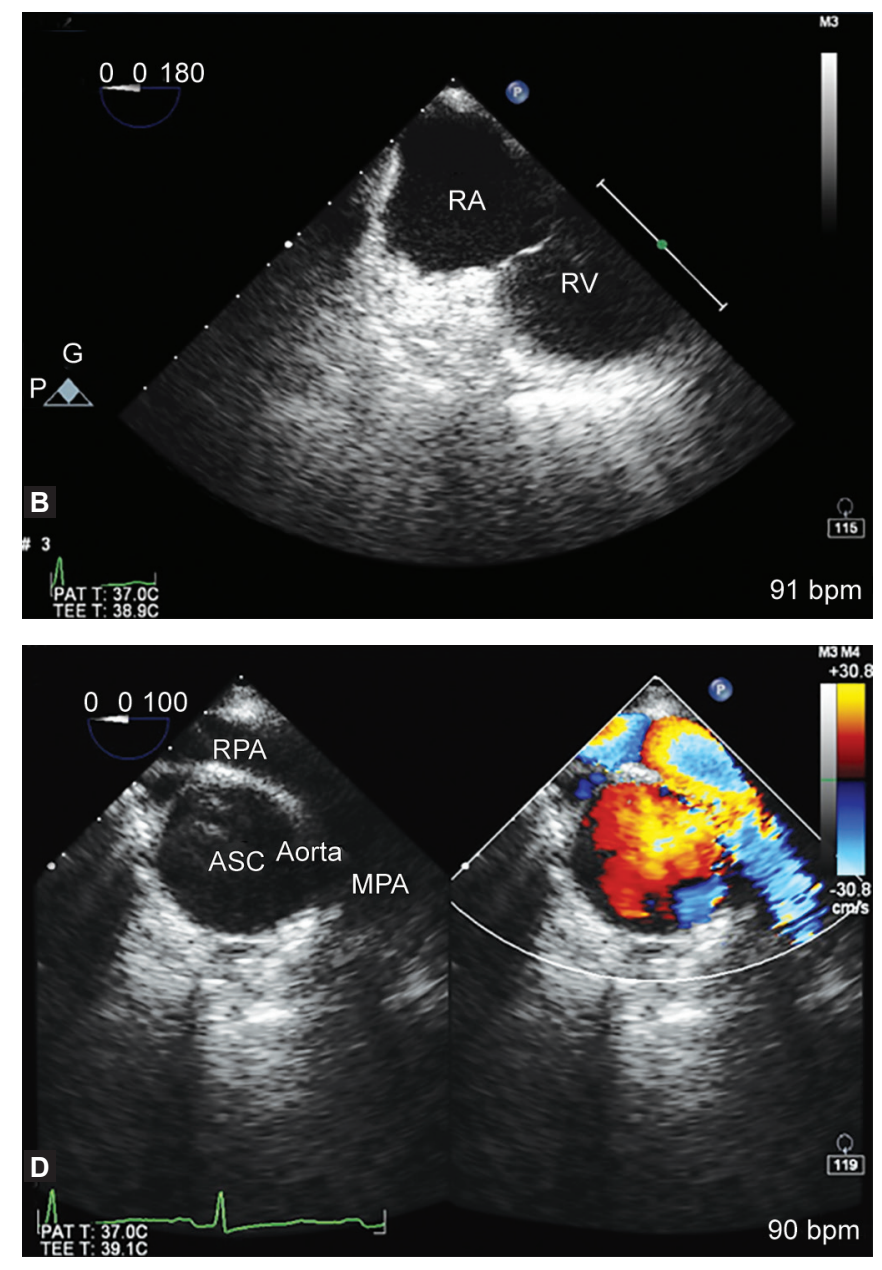

Figs 7A to D: (A) Mid-esophageal bicaval view showing empty right atrium with SVC and IVC communicating with right atrium; (B) Mid-esophageal echocardiography with focused right atrium showing no mass in right atrium; (C) Transgastric IVC long axis view showing no filling defect in IVC; (D) Ascending aortic short axis view showing right pulmonary artery and main pulmonary artery with no filling defect and turbulence.

presentation and it is rarity, leiomyoma with intracardiac extension can be misdiagnosed as either thrombus, myxoma or renal cell carcinoma with intra cardiac extension. On echocardiography leiomyomatosis should be suspected when a long, serpentine, round and nonadherent mobile mass is noticed extending from the IVC into the right atrium. Intravenous leiomyoma is a benign smooth muscle cell tumor of uterine origin that may grow into the pelvic veins and the inferior vena cava. ${ }^{1-4}$ Uterine leiomyomatosis is a systemic leiomyoma that metastasizes beyond the uterus.The principle of surgery is to remove the intracardiac mass to prevent sudden 
death, and radical resection of the involved organ to prevent recurrence. During the retrieval of the intracaval tumor, it may break and result in embolism and sudden cardiac collapse.

Intraoperative TEE is a valuable tool for confirming the underlying diagnosis and to guide the surgeon during the surgery. On TEE examination, an important and peculiar feature of leiomyoma with IVC and cardiac extension is the lack of adhesion to the wall of cardiac and venous structures. ${ }^{5}$ Renal cell carcinoma is a malignant tumor which invade IVC and propensity to extend into right heart. ${ }^{6}$ The accurate assessment of the distal extension of leiomyoma into the inferior vena cava and the right atrium is crucial before surgical resection. The most valuable function of TEE in the intraoperative period is the continuous monitoring of the tumor during manipulation. It enables rapid recognition of tumor embolization, which allows earlier notification to the surgeons and commencement of management even before hemodynamic instability develops. ${ }^{7,8}$ In the intraoperative period, TEE also help us in the assessment of biventricular function, volume status and confirming the re-establishment of intracaval blood flow. In our case report, the patient was fully heparinised and placed on partial CPB with suction catheter in the right atrium for venous drainage from the coronary sinus and the IVC. The tumor was then retrieved with gentle pull from the suprarenal and infrarenal IVC through a supra renal venacavotomy, TEE also helped to confirm complete tumor removal, and no residual tumor fragments in the right atrium and the IVC.

\section{CONCLUSION}

Intravenous leimyomatosis is a rare condition of the uterus that may involve tumor extension into the IVC and the right side of the heart. TEE can be helpful during central line insertion to avoid dislodgement of the tumor by the guidewire. TEE is also helpful to rule out the occurrence of embolic events during the intraoperative period through continuous monitoring. It also helps confirm re-establishment of intracaval blood flow and complete tumor extraction from the IVC and the right side of the heart.

\section{REFERENCES}

1. Nam MS, Jeon MJ, Kim YT, Kim JW, Park KH, Hong YS. Pelvic leiomyomatosis with intracaval and intracardiac extension: a case report and review of the literature. Gynecol Oncol 2003;89:175-180.

2. Norris HJ, Parmley T. Mesenchymal tumors of the uterus. V. Intravenous leiomyomatosis. A clinical and pathologic study of 14 cases. Cancer 1975;36:2164-2178.

3. Lam PM, Lo KW, Yu MY, Wong WS, Lau JY, Arifi AA, Cheung $\mathrm{TH}$. Intravenous leiomyomatosis: two cases with different routes of tumor extension. J VascSurg 2004;39: 465-9.

4. Harris LM, Karakousis CP. Intravenous leiomyomatosis with cardiac extension: tumor thrombectomy through an abdominal approach. J VascSurg 2000;31:1046-1051.

5. Lou Y.F., Shi X.P., Song Z.Z. Intravenous leiomyomatosis of the uterus with extension to the right heart. Cardiovasc Ultrasound. 2011;9:25.

6. Allen G, klingman R, Singh A. A transesophageal echocardiography in the surgical management of renal cell carcinoma with intracardiac extension. J Cardiovascular surg 1991 Nov-Dec; 32(6): 833-836.

7. Komanapalli CB, Tripathy U, Sokoloff M, Daneshmand $\mathrm{S}$, Das A, Slater MS. Intraoperative renal cell carcinoma tumor embolization to the right atrium: incidental diagnosis by transesophageal echocardiography. AnesthAnalg. 2006;102:378-379

8. Sharma V, Cusimano RJ, McNama P, Wasowicz M, Ko R, Meineri M. Intraoperative migration of an inferior vena cava tumour detected by transesophageal echocardiography. Can J Anaesth. 2011;58:468-470 